\title{
The Inheritance and Innovation of Sketch Art Against the Background of Modern Cultural Construction*
}

\author{
Wenjuan Huang \\ Nantong Institute of Technology \\ Nantong, China
}

\author{
Zhifang Shi \\ Hand Drawing Education of Jingcheng Design \\ Huainan, China
}

\begin{abstract}
Sketch is a hand-painted expression with a long history, with rapid performance, rich graphics, strong modeling and other characteristics. The sketch art has created a large number of excellent works through the inheritance of several generations of artists for hundreds of years, and then gradually become one of the ways of expressing works in the art and design industries. With the continuous integration of Chinese and Western cultures, sketch art has undergone new development and changes. Under the background of the cultural construction of "concentration and innovation" put forward in the 13th Party Congress of Jiangsu Province, this paper bases on the traditional sketch method, explores the innovative research of sketch art from innovation of ideas, innovation in form of expression and innovation in art application.
\end{abstract}

Keywords—sketch; inheritance; innovation

\section{INTRODUCTION}

"Sketch" originated in Europe in the 18th Century. It is a method of painting expression to train the comprehensive ability of modeling. It shows aesthetic value, attaches the artist's thought and emotional characteristics, and shows extraordinary artistic charm. It is the application of overall analysis and the refinement and development of general expression in the subject of sketch. Van Gogh, Rodin, Picasso and other artists have left very expressive sketch works of art. In China's famous artists, Ye Shanyu, Huang Gang and Li Shinan set up the peak of sketch art, continuing to this day. With the development of art, sketch has gradually become an independent form of practical art. Its fast and direct picture expression has been recognized and valued by the art industry and design circles.

\section{THE INHERITANCE OF SKETCH ART}

Sketch trains people's ability to quickly capture the environment and modeling characteristics. Facing with real scene, painters record form, characteristics and environmental relations of the scene with refined lines in a short period with

*Project source: Research on the Inheritance and Innovation of Sketch Art under the Background of Jiangsu Cultural Construction, the project of Philosophy and Social Sciences Research Foundation of Jiangsu Colleges and Universities (Special topic), No. 2017SJB1283; Innovation Research of Teaching Mode in the Course of Architectural Hand-painted Performance in Informatization, Nantong Institute of Technology Teaching Reform and Construction Project, No.: 2017NITJG10; the Qinglan training project of outstanding young backbone teachers from Jiangsu Colleges and Universities in 2018 . the help of certain performance tools to paint realistically. Using the transformation of point, line and plane, the harmonious configuration of density and darkness, the hierarchical relations of scene objects are transformed and connected to express the attributes and characteristics of picture objects. Among them, the sketch form of pen and pencil is the most common. The reasonable organization of lines, the density of lines, the thickness of lines, the speed of lines and the blank of the picture have a great impact on the effect of the picture.

The traditional theory and practice of sketch art are very fruitful. For example, Yi Xiaolei pointed out the irreplaceable fresh vitality and flexibility of sketch art in his article Valuable Intuition - the Importance of Sketch in Art Creation. Wen Peng summarized the opportunities to be discovered in sketch with the rapid development of society in his article Analysis of the Crisis and Development Opportunity of Sketch Art. In the article Seen from Small Things - Artistic Value and Emotional Expression in Sketch, Sun Zhe put forward to find the artistic value, status, new development direction of sketch. Xia Keliang's monograph Pen Sketch of Architectural Landscape has influenced a lot of painting learners, as well as Liu Yuxing's Pen Landscape Sketch and other artistic works and courses. In the process of practical creation of sketch art, the works show the artistic development state of documentary, literature and art, and regional characteristics. For example, in 2015, the Art Department of the Ministry of Culture and the Chinese Art Association jointly sponsored the "Chinese Art Taihang Forum - the Tradition and the Present Significance of Sketch, and proposed the contemporary art responsibility and culture continuation of sketching. In 2013, Zhao Ming, 18, held the Sketch Decade - Zhao Ming's Works Exhibition at the Academy of Fine Arts of Hunan Normal University. The exhibition has attracted wide attention from the art circles, reflecting the inheritance spirit of Chinese sketch art Guangzhou has begun to hold sketch exhibitions since 2011, which shows the government's attention and encouragement to sketch art. Professor Cao Yiqiang of the Chinese Academy of Fine Arts has held a Cao Yiqiang's Sketch Exhibition at the Nanyi Art Museum to show more than 60 sketches he has created in recent years. He showed the recording and development significance of sketch to society. The sketch exhibition, Capture Moments - Works of Ye Shanyu and Chen Danqing, sponsored by the National Grand Theatre, shows the social importance and development peak of the sketch art in China. 
It can be seen that the inheritance and development of sketch art is the responsibility and obligation of contemporary artists and the carrier of Chinese culture. In November 2017, the National History and Culture City Research Center of Tongji University and the Chinese Hand-painted Art Research Institute joined hands with modern hand-painted art teachers, such as Zhou Feng, Shi Zhifang, Li Lei, Chen Lifei and Huang Ge, to carry out the public welfare activity "Hand Painting \& Travel" in Hongcun Village. Like many star-rated ancient tourist villages, the ancient architectural complex of Hongcun Village is becoming more and more commercialized in her tourist development. It is facing new problems, such as the old buildings being out of repair and the protection of ancient dwellings. The purpose of the activity was to show the ancient architecture complex of Hongcun Village, which has a history of nearly 1000 years, to all walks of life through sketch art works. It can show the artistic value of the ancient dwellings and help the residents obtain commercial value. With one village, 112 painters and 158 ancient dwellings of Ming and Qing Dynasties and more than 300 selected works, the artistic, communicative and practical characters of sketch are fully displayed. The interactive effect of activity and sketch achievements are better. Participants recorded time, told stories and showed the splendor of ancient architectural art with sketches. Sketching techniques integrates with ancient architecture heritage. Artists used brushes to retain the fading ancient village culture and action to call for the protection of ancient village culture.

Throughout the existing results, we tend to training of sketch from nature. We have less research on its inheritance. Even if some of the views put forward are still in the theoretical guidance level. Professor Ye Shaoyu, a famous Chinese painter, appraised the art of sketch as "Not a small skill, not a small path". It can be seen that carrying forward the art and culture of sketching and expressing the national spirit need the joint excavation, protection and inheritance of the society and the art circles.

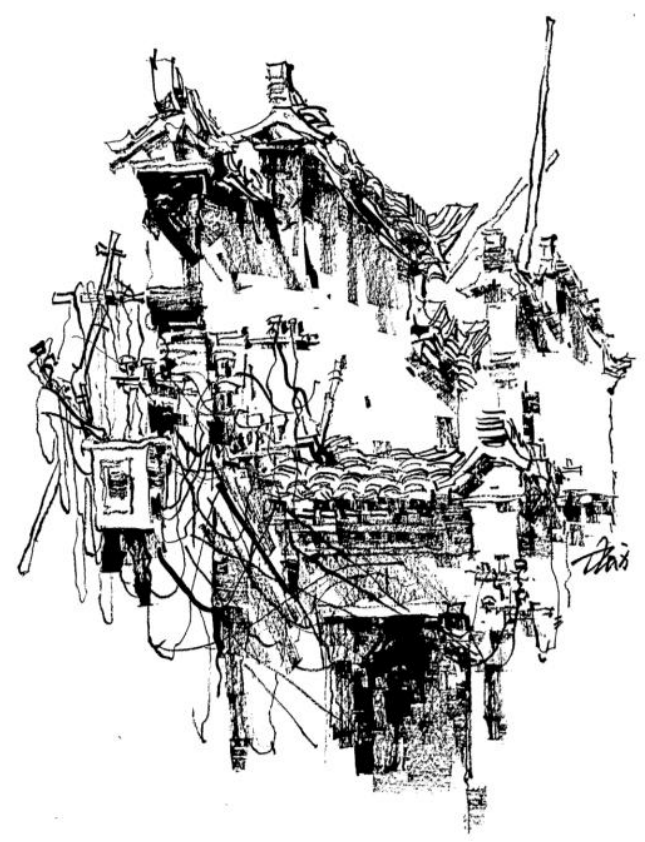

Fig. 1. Pen sketch: Ancient architecture in Hongcun Village - Shi Zhifang.

\section{INNOVATION OF SKETCH ART}

If sketch art follows a single way of inheritance, display and creation, it cannot keep pace with the times of creative creation. It will be lack of innovative ideas in the cultural era, neglect the operability of cultural and artistic value expression, and eventually fade out of people's vision. With the continuous integration of Chinese and Western cultures, conceptual changes and cross-border art trends of thought, the art of sketch has undergone new development and changes. Based on traditional methods of sketch, the innovation of sketch art concept can be used to make sketch close to modern art life and creation. Combined with the characteristics of the times and cultural performance, the innovation of sketch art in forms of expression is the way to develop the art of sketch with the times and to develop new markets.

\section{A. Innovation of Ideas}

With the vigorous development and promotion of cultural format innovation in recent years in China, we put forward to inherit and carry forward excellent traditional culture with regional characteristics, striving to create more cultural boutique instructions. Supported by the policies of various circles of the state, sketch art should be closely combined with social needs and policy orientation from the angle of creation. We should break through the traditional, realistic and linebased recording method, which can be exaggerated, distorted and abandoned in the process of creation. Around the characteristics of China's cultural construction and the characteristics of the times, we can make sketch art creation with high-quality culture.

In recent years, digital art, as a leading industry in the information age, has developed rapidly. It is based on all kinds of cultural and artistic resources and powered by people's spiritual creativity and skills. Considering from the innovative 
idea, in the scientific and technological trend society characterized by digitalization and modernization, modern sketch can use digital creative platform to expand thinking, and combine digital creativity to create, disseminate and trade cultural value. We can expand the unique expressive tension and application scope, excavate and present concept innovation of sketch works of art, creating excellent works with artistic value and social spirit.

\section{B. Innovation in Form of Expression}

The form of traditional sketch is simple and varied. Being simple is because sketch gives priority to black and white in the performance process, such as pen sketch, pencil sketch and charcoal sketch. Being varied is because it has rich contents of sketch, such as scenery, scenes, characters and animals. After artists has inherited the main points of sketch art, with the cross-border of tools and artistic expression, modern sketch art can embody innovation from the comprehensive use of forms of expression.

1) Light color sketch: Light color sketch is based on pen sketch and pencil sketch. It takes watercolor as a coloring tool and creates according to reality. Watercolor painting has a long history and is more common. Light color sketch is refined and innovated from sketch art and watercolor painting by artists in recent years in order to better reflect the color relationship and painting mood. It is a fast color sketch expression form favored by artists. On the basis of sketch, the light color relationship can not only reflect the rapid expression and detailed perspective of sketch, but also show the softness and color of watercolor, and show the comprehensive characteristics of sketch and watercolor. For example, the hand-painted art creator Shi Zhifang's lightcolored sketch work Ancient Architecture and Red mainly shows traditional architecture, adopts pen sketch as the basis of line draft, follows the perspective principle of ancient residential buildings and object proportion relationship, outlines the architectural features and details of Hui-style residential buildings, and uses pen and ink color to show the basic light and shadow effects and contrast with light and shade, then uses simple light colors to restore the building's color relationship. It can enhance the artistic interest of the picture. The red lantern at the gate has reflected the artistic beauty of the picture, becoming the finishing touch in the overall picture.

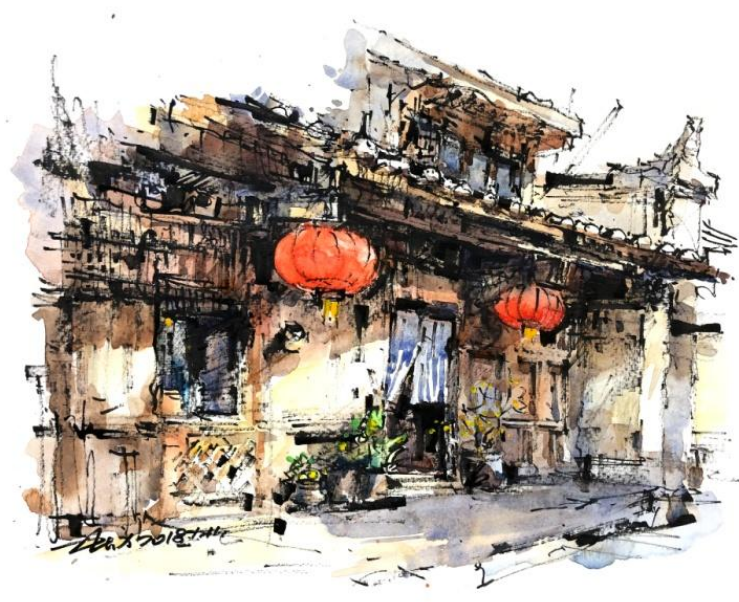

Fig. 2. Light color sketch: Ancient architecture and red - Shi Zhifang

2) Marker sketch: Marker is one of the hand-painted tools for rapid expression in the West. Marker sketch is a new form of sketch. It has been introduced into China for only ten years. Based on the line modeling of the picture, with the help of marker, a new type of pen tool, it can perform well in the fields of sketch, design, illustration, draft and so on. Marker sketch is a practical art with independent aesthetic value. It is a relatively new form of expression compared with the traditional sketch art. Its greatest function is to make a leapforward change in the sketch from simple appreciation to application. With the continuous integration of Chinese and Western cultures, in recent years with the market and industry demand, marker sketch has a new development and change. It has summed up a certain theoretical and practical basis, showing a form-personalized and innovative development. For example, Huang Wenjuan's marker sketch Campus of Nantong Institute of Technology is based on a smooth and lively line picture. The sketch chooses a large campus scene with a fountain as the center. The pen shows the perspective of the scene and the proportional relationship between the buildings. The painter used the sensitive and rigorous marker coloring technique, paid attention to the light and shade effect of natural light sources and the combination of similar colors. The overall picture presents a variety of creative and rapid expression of artists and designers.

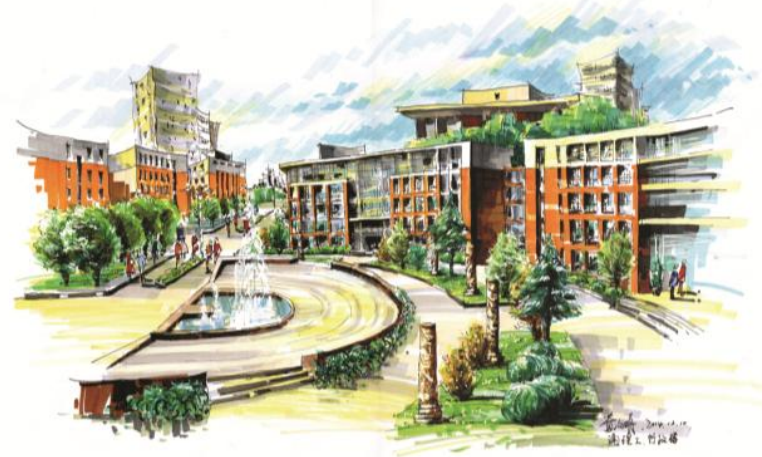

Fig. 3. Marker sketch: Campus of Nantong Institute of Technology - Huang Wenjuan. 
3) Rice paper sketch: Rice paper is a traditional Chinese painting and calligraphy paper with a history of more than 1500 years. It is easy to preserve, not brittle and not fading for a long time. A large number of rare ancient books, calligraphy and paintings are preserved by rice paper, and the picture effect is still the same. We can see the reputation of rice paper as "millennium life paper". In the process of sketch innovation, some modern artists attempted to use rice paper for pen sketch. On the basis of rice paper's unique penetration properties, painters control the proportion of water and ink and use marker or watercolor for color rendering, resulting in a Chinese charm of "five-color ink". Completing with one stroke, it has thick and thin color, texture, clear ink charm and distinct gradation. The special rendering effect of rice paper sketch gives a kind of artistic expression which can be achieved quickly and slowly.

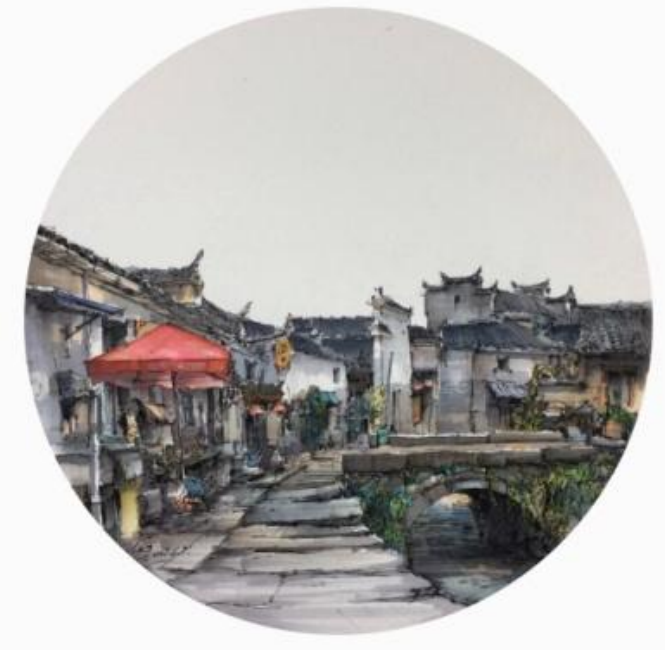

Fig. 4. Rice paper sketch: Campus of Nantong Institute of Technology - Shi Zhifang.

\section{Innovation in Art Application}

Art should have the value of expression, appreciation, collection, dissemination and application, and sketch art is no exception. In recent years, with the growth of social individualized demand, the national industrial policy encourages the art industry to develop toward high-tech products. As a result, the domestic art creation performance market develops rapidly, and product output continues to expand. On November 29, 2016, the State Council issued the National Strategic Emerging Industries Development Plan for the 13th Five-Year Plan, which proposed that China should form a cultural and creative industry development pattern with cultural guidance, artistic development, advanced technology and complete chain by 2020. The 13th Party Congress of Jiangsu Province in 2016 clearly put forward the concept of "cohesive innovation". It put innovation at the core of the overall development, made it clear that we should vigorously develop creative cultural industries, promote the innovation of cultural formats, further inherit and carry forward the excellent traditional culture with regional characteristics, and create more cultural products.
It can be seen that sketch art has greater display stage and development space in the innovation of application value. For example, Masao Yamada's 15 Minutes Travel Postcard Sketch, Wuxi City's the Postcard Design Competition in 2013, the success of students of Shenyang Normal University Handpainted Campus Postcard and various types of city postcards, handbags, stationery, refrigerator stickers and letterhead are all dependent on the innovative development and application value of sketch art in cultural industry. For example, Huang Wenjuan's modern sketch work "Yangyang Rich Postcard" is based on the traditional pen line sketch. The painter used the innovation of marker in expression and expressed the creative performance according to the needs of the enterprise. The work is authorized to be patented and used practically by Jiangsu Postal Savings on delivery advertisement.

The application innovation of sketch art should be based primarily on the market demand for artistic innovation, improve the creative ability of the creator, create a unique artistic image, show the application innovation of sketch art outside the realistic record, make the value transformation of sketch art in the literary creation industry rise abruptly, achieve results, and realize the mutual integration and innovation of artistry and application.
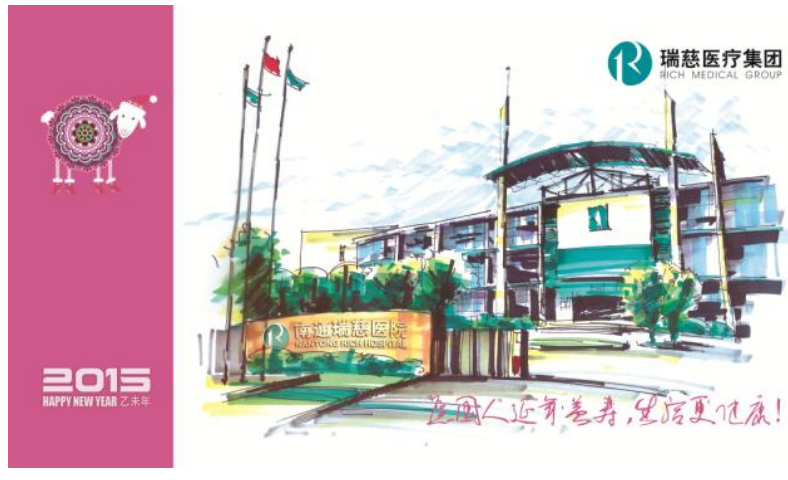

Fig. 5. Sketch application: Yangyang Rich Postcard - Huang Wenjuan

\section{CONCLUSION}

As a form of traditional painting, sketch is the basis of various forms of art. It represents comprehensive quality of artists and designers. The creation of modern sketch art meets the needs of the times, inherits the expression of sketch, tries to use new materials and tools with the leading of new idea in sketch art, explores the applied innovation of sketch art, promotes the organic integration of sketch art and new format, and enriches the feasibility of new creativity, new format and new development in the cultural construction in China, enhances the new level of sketch art, realizes the visual art value and practical application value of sketch, thus promotes the transformation and progress of China's cultural industry.

\section{REFERENCES}

[1] Jiang Bin. New Ideas, New Tools, New Methods, New Paintings. Art Observation, 2010, 01: 78-80

[2] Cheng Qingshi. Bearable Heaviness of Marker - When Marker encounters Xia Keliang. Architecture and Culture, 2012, 03: 122-123. 
[3] Huang Wenjuan. Sketch Methods of Architecture for Design Majors. Research on Private Higher Vocational Education, 2014, 10.

[4] Huang Wenjuan. Architectural Sketch. Changchun: Northeast Normal University Press, 2014.

[5] Wen Peng. Brief Analysis of the Crisis and Development Opportunities of Sketch Art. Art Education Research, 2011, 12: 176-177.

[6] Richard McGary, Greg Madson. Charm of Marker. Shanghai: Shanghai People's Art Publishing House, 2015.

[7] Shi Zhifang. Retention of Memory. Shanghai: Tongji University Press, 2017. 